\title{
The Puzzle of quasi prima 'almost before' and quasi dopo 'almost after'
}

\author{
Patrícia Amaral \\ Stanford University
}

\author{
Fabio Del Prete \\ Università degli Studi di Milano \\ Stanford University
}

\section{A Puzzling Asymmetry}

This paper focuses on a puzzling asymmetry which arises when the Italian approximative adverb quasi 'almost' modifies the temporal connectives prima 'before' and dopo 'after'. By looking at naturally occurring data, we observe that $A$ quasi prima che $B$ 'A almost before B' can be used to convey that an Aeventuality occurs early, only a little bit after a B-eventuality occurs. Consider the sentences $(1 \mathrm{a}, \mathrm{b})$, both adapted from examples taken from the web:

a. Le pere Cocomerine vanno consumate subito, al momento della raccolta, quasi prima che cadano.

'Cocomerine pears have to be consumed soon, at the time of their picking, almost before they fall.'

b. [Context: The soccer player Tommaso Rocchi plays two matches at the beginning of the Olympic Games. Then he has to discontinue his participation in the games because of an injury.]

L'avventura olimpica di Tommaso Rocchi è finita quasi prima di cominciare.

'The Olympic adventure of T. Rocchi ended almost before it started.'

Sentence (1a) means that Cocomerine pears have to be consumed soon, shortly after they have been picked. Sentence (1b) means that the adventure of T. Rocchi at the Olympics ended early, shortly after it started.

A sentence of the form A quasi dopo che B 'A almost after B', however, cannot be used to convey that an A-eventuality occurs late, only a little bit before a B-eventuality. Consider the constructed examples $(2 a, b)$ :

(2) a. ? Le pere Cocomerine cadono quasi dopo che è passato l'autunno.

'Cocomerine pears fall almost after the autumn has passed.'

b. [Context: The soccer player Tommaso Rocchi plays two matches at the very end of the Olympic Games to replace another player who suffered an injury.]

? L'avventura olimpica di Tommaso Rocchi è cominciata quasi dopo che sono finite le Olimpiadi.

'The Olympic adventure of $T$. Rocchi started almost after the Olympics ended.' 
Sentence (2a) cannot mean that Cocomerine pears fall late, shortly before the autumn has passed. Analogously, (2b) cannot mean that the Olympic adventure of T. Rocchi started late, shortly before the Olympics ended. Both $(2 a, b)$ are unacceptable on these readings. For some native speakers, however, sentence (2a) can be used to express the proposition that Cocomerine pears fall shortly after the end of the autumn. In section 4, we return to such readings of quasi dopo, in which it seems that quasi contributes the meaning of the modifier poco 'a little bit'.

We propose a solution for this puzzle that relies on an analysis of quasi as a scale-sensitive adverb and on an asymmetric semantic account of prima and dopo: prima has the meaning of the temporal comparative 'earlier', while dopo denotes a binary relation of temporal succession between events. We show that our account of quasi prima reveals a more general pattern of interpretation found when quasi modifies a comparative.

\section{Scalarity of quasi}

We assume that quasi conveys approximation to a limit along a scale of a certain dimension, and that it associates with a focused constituent, like English only (cf. Rooth 1985). The scalarity of quasi is shown by the examples below, in which quasi modifies temporal phrases: in (3a), quasi modifies a locational time adverbial (alle tre 'at three pm') and in (3b) and (3c) it modifies the directional time adverbials fino a mezzanotte 'until midnight' and dal 2000 'since 2000', respectively.
a. Gianni è arrivato quasi alle tre.
'Gianni arrived at almost three pm.'
b. Leo ha fumato quasi fino a mezzanotte.
'Leo smoked almost until midnight.'
c. Viviamo qui quasi dal 2000.
'We have lived here almost since 2000.'

In isolation, (3a) is interpreted as implying that it was not yet three pm when Gianni arrived (i.e. the time of Gianni's arrival precedes three $\mathrm{pm}$ ), and that the time at which Gianni arrived closely approximates three pm (from the left, in a left-to-right representation of the ordering of times from earlier to later). This situation is diagrammed in Figure 1.

Figure 1

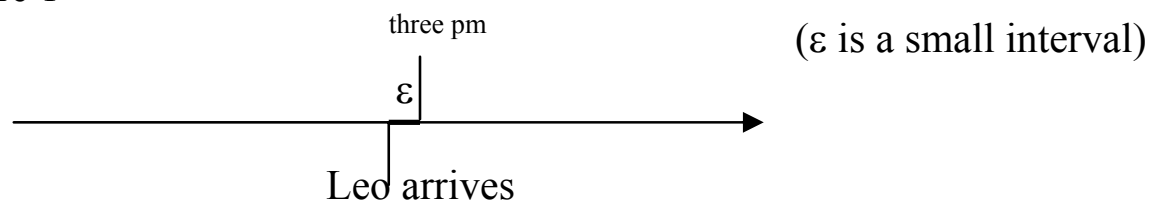


The interpretation of ( $3 b)$ is that Leo went on smoking up to a point in time which was shortly before midnight. The interpretation of (3c) is that the state of our living here stretches backward up to a point in time which is shortly after 2000 . These interpretations are represented in Figures 2 and 3, respectively. As shown by the diagrams, the interpretation of quasi fino is the mirror-image of the interpretation of quasi $d a$.

Figure 2

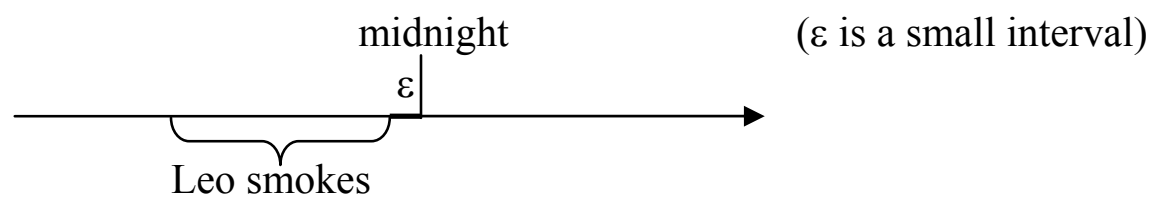

Figure 3

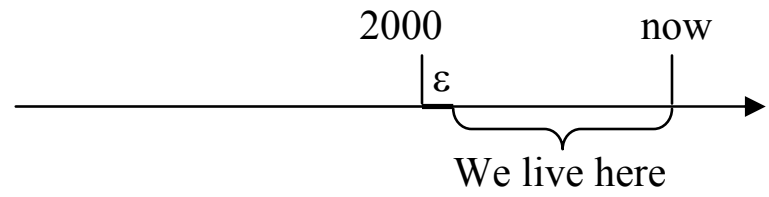

The semantic pattern that arises from the interpretation of (3a-c) can be described as follows: when quasi modifies a temporal phrase with the locational preposition $a$ 'at' or with the directional preposition fino 'until', we obtain an effect that we can describe in intuitive terms as approximation to a limit from the left. The limit is the temporal location in (3a) and it is the temporal goal introduced by the complement of fino in (3b). On the other hand, when quasi modifies a temporal phrase headed by $d a$, as in (3c), the effect is approximation to a limit from the right; the limit is the temporal source introduced by the complement of the temporal connective. While in $(3 \mathrm{a}, \mathrm{b})$ the relevant ordering of the temporal alternatives is from earlier times to later times, with quasi da the ordering is from later times to earlier times, so that we have the reversed temporal scale. We hypothesize that approximation to the limit from the left or from the right is a direct consequence of the semantics of the temporal connective modified by quasi. Approximation from the left is due to the inherent directionality of fino, which requires a movement along the time axis from the past to the future, i.e. from the left to the right, and approximation from the right is a direct consequence of the inherent directionality of $d a$, which requires a movement on the time axis from the future to the past, i.e. from the right to the left. ${ }^{1}$ The locational temporal preposition $a$ does not encode a directionality, hence the ordering of the temporal scale is given by the prototypical direction of time as we experience it, namely from the past to the future.

\footnotetext{
${ }^{1}$ For a full-fledged analysis of sentences with quasi fino 'almost until' and quasi da 'almost since', in which the direction of approximation to the limit is obtained as a direct consequence of the semantics of the temporal connectives fino and da, see Amaral and Del Prete (2009).
} 


\section{Analysis of quasi}

Informally, we analyze the meaning contribution of quasi as involving the following components: ${ }^{2}$

(A) Quasi entails negation of the smallest sentence in which the adverb occurs (we call it the prejacent, following Horn 1996), as shown in (4). For our present purposes, we make the simplifying assumption that quasi applies to a sentential argument, a constituent of which is focused. ${ }^{3}$

$$
\text { Quasi(S) entails not(S) }
$$

To exemplify, for sentence (3a) above this entailment is as follows:

$$
\text { Quasi(Leo è arrivato alle tre) entails not(Leo è arrivato alle tre) }
$$

We assume that the negation contributed by quasi is focus-sensitive, and is not the simple logical connective $\neg$ : it does not simply deny the truth of the prejacent, but, more specifically, it denies the truth of the prejacent for the particular value of the focused constituent. For example, sentence (3a) entails that Leo arrived and that Leo's arrival occurred at a time that is not three pm.

(B) The meaning of quasi is such that the focused constituent, possibly with the support of the conversational context, must provide two ingredients: (a) a scale $S$, i.e. a set of alternatives (the domain of $S$ ) with a linear order on it; ${ }^{4}$ (b) a value on

\footnotetext{
${ }^{2}$ The negative implicatum in (A) and a component of close approximation that subsumes (B) and (C) correspond to the "polar" and the "proximal" component, respectively, into which Sevi (1998) and Horn (2002) analyze the meaning of almost. In our account, we treat the meaning components (A)-(C) as entailments of almost and do not assume any difference in the role they play in the interpretation of the adverb. However, we are aware that the properties of these components are complex, as shown by their behavior with respect to the standard tests for different types of implications. For discussion of different proposals, see Horn (2002) and Amaral (2007, to appear).

${ }^{3}$ Throughout this paper, we adopt the convention of marking focus on a constituent by means of boldface. For example, when we write at three pm we intend to refer to a focused instance of the time adverbial at three $\mathrm{pm}$.

${ }^{4} \mathrm{~A}$ two-place relation $\mathrm{R}$ is said to be a linear order if it satisfies the following conditions (see Landman 1991: 84):

(c1) $\quad \forall x \mathrm{R}(x, x)$

(c2) $\quad \forall x \forall y[[\mathrm{R}(x, y) \wedge x \neq y] \rightarrow \neg \mathrm{R}(y, x)]$

(c3) $\quad \forall x \forall y \forall z[[\mathrm{R}(x, y) \wedge \mathrm{R}(y, z)] \rightarrow \mathrm{R}(x, z)]$

(reflexivity)

(antisymmetry)

(c4) $\quad \forall x \forall y[\mathrm{R}(x, y) \vee \mathrm{R}(y, x) \vee x=y]$

(transitivity)

(connectedness)

For our purposes, it does not matter whether the relation which orders the focus-alternatives is reflexive. In this paper, we adopt the notation ' $<_{s}$ ' to refer to the linear order defining a scale $S$, instead of adopting the notation ' $\leq_{s}$ ', thus suggesting that we consider strict (i.e. non reflexive) linear orders. However, our choice is uniquely due to practical convenience, and nothing essential in our proposal hinges on the assumption that the relation ordering the alternatives is a strict linear order rather than a reflexive linear order.
} 
$S$, which we call limit point. ${ }^{5}$ For example, in most circumstances an utterance of the focused PP alle tre 'at three pm' will provide a scale intuitively consisting of temporal locations linearly ordered from earlier to later, and it will always provide a value from that scale, i.e. the temporal location three $\mathrm{pm}$.

(C) A sentence 'Quasi $(\varphi)$ ' entails a related sentence $\varphi$ ', where $\varphi$ ' expresses a proposition in which existential quantification is made over alternatives to the focused constituent of $\varphi$ which are ranked lower than the limit point and situated close to it on the relevant scale $S$. How much an alternative must be close to the limit point in order to be taken into account for the quantification is a contextdependent issue. This entailment relation is schematically given as follows:

$$
\text { Quasi(... } \left.\alpha \ldots) \text { entails (for some } d<_{S}[[\alpha]] \& \operatorname{close}(d,[[\alpha]])\right)(\ldots d \ldots)
$$

In our formal analysis, we assume an event semantics framework. Verb predicates, as well as adverbial modifiers, project an argument position for eventualities, which is abstracted over by the $\lambda$-operator (Davidson 1967; Parsons 1985, 1990; Landman 2000). Adverbial modification is handled by means of predicate intersection, i.e., the $\lambda$-abstracts $\lambda e_{\mathrm{E}} \mathrm{V}(\ldots e \ldots)$ and $\lambda e_{\mathrm{E}} \cdot \operatorname{Adv}(\ldots e \ldots)$, which correspond to the verbal and to the adverbial projection respectively, are combined so as to yield the derived abstract $\lambda e .[\mathrm{V}(\ldots e \ldots) \wedge \operatorname{Adv}(\ldots e \ldots)]$. A default operation of existential closure maps a $\lambda$-abstract $\lambda e . \varphi(e)$ onto the existentially quantified formula $\exists e \varphi(e)$. Temporal phrases are adverbial modifiers, and are treated accordingly as intersective modifiers. As for quasi, we assume that it is a cross-categorial modifier which takes a focused constituent $\alpha$ as argument and yields the logical conjunction of two elements: the negation of the proposition resulting from the application of the semantic value of $\alpha$, and a proposition resulting from the application of a lower ranked alternative to the semantic value of $\alpha$. We assume a semantics for focused expressions along the following lines: the semantic contribution of a complex $[\alpha]$ F consisting of an expression $\alpha$ ( $\alpha$ being of some syntactic category) and a focus [ ]F on $\alpha$ is analyzed as an ordered pair $\left\langle\alpha^{\prime}, S_{\alpha^{\prime}}\right\rangle$ in which the first coordinate is the semantic value of $\alpha$ and the second coordinate is a set of alternatives to $\alpha^{\prime}$, all of the same semantic type as $\alpha^{\prime}$ and ordered according to some linear relation. We refer to such ordered pairs as f-pairs. On this semantics, the specific contribution of focus

\footnotetext{
5 In order for an object $x$ on a scale $S$ to possibly count as a limit point on $S$, the following condition must be met (Condition on Limit Points):

(CLP) $x$ must contribute a value $v$ to the truth-condition of the sentence which is definite enough for a speaker to be able to recover a value $v^{\prime}$ which is different from $v$ but close to $v$.

This condition explains why e.g. quasi qualche 'almost some' is not acceptable: given that the semantic value $x$ of qualche is the existential quantifier (corresponding to 'at least one'), $x$ does not contribute any definite (numerical) value to the truth-conditions of a sentence Qualche $A \grave{e} P$ 'Some A is P', which indeed can be true in virtue of any number of As being P. Given that no definite numerical value is contributed to the truth-conditions by qualche, it follows that a speaker could not recover a different and close value and use it in the interpretation of Quasi qualche $A \grave{e} P$ 'Almost some A is P'.
} 
can be analytically identified with the set of alternatives to the semantic value of the expression bearing focus. We do not see the linear order on the alternatives as part of the semantic contribution of focus, but rather as contextually determined. Given the above assumptions, we propose the interpretation for quasi given in (7). On this interpretation, quasi takes two arguments: the first argument is an $f$-pair, and the second argument is the semantic value of the part of the sentence which is not in focus. The first coordinate of the $f$-pair is the semantic value of the focused constituent $x$ and its second coordinate is the set of alternatives to the semantic value of $x$ provided by focus, that are ordered in a contextually-relevant way.

$$
\begin{aligned}
\text { [[ quasi ]] }= & \lambda<P_{<\gamma, t>}, S>. \lambda x_{\gamma \cdot} \neg P(x) \wedge \exists Q Q_{<\gamma, \downarrow} \in S\left[Q<_{S} P \wedge \operatorname{close}_{S}(Q, P)\right. \\
& \wedge Q(x)]^{6}
\end{aligned}
$$

We provide an example of how our analysis works by applying it to sentence (3a), repeated below as (8). The constituent in focus is the time adverbial alle tre. Accordingly, the scalar alternatives which are taken into account in computing the truth-conditional meaning of the sentence are objects of the same semantic type as alle tre. The semantic values of the unfocused alle tre and the focused alle tre are given in (9a) and (9b), respectively. In (10) we give a schematic representation of the scale $S$ which is relevant to the interpretation of the sentence. Notice that the relation $<_{S}$ underlying this scale is such that, for any alternatives $\left(\lambda e . \tau(e) \subseteq t_{1}\right)$, $\left(\lambda e . \tau(e) \subseteq t_{2}\right)$ in the domain of $S,\left(\lambda e . \tau(e) \subseteq t_{1}\right)<_{S}\left(\lambda e . \tau(e) \subseteq t_{2}\right)$ if and only if $t_{1}$ temporally precedes $t_{2}{ }^{7}$ The main steps of the semantic composition are given in (11a-c). The final truth-conditions are derived from (11c) by the default operation of existential closure, and are reported in (12).

(8) Gianni è arrivato quasi alle tre.

a. $[[$ alle tre $]]=\lambda e . \tau(e) \subseteq 3 \mathrm{pm}$

b. $\quad[[$ alle tre $]]=<\lambda e . \tau(e) \subseteq 3 \mathrm{pm}, S>$

$(\lambda e . \tau(e) \subseteq 12 \mathrm{pm})<_{S}(\lambda e . \tau(e) \subseteq 1 \mathrm{pm})<_{S}(\lambda e . \tau(e) \subseteq 2 \mathrm{pm})<_{S}(\lambda e . \tau(e) \subseteq 3 \mathrm{pm})$

a. $\quad[[$ quasi alle tre $]]=\lambda e_{\mathrm{E}} . \neg \tau(e) \subseteq 3 \mathrm{pm} \wedge \exists Q_{<\mathrm{E}, \mathrm{t}>} \in S\left[Q<_{S}(\lambda e . \tau(e) \subseteq\right.$ $\left.3 \mathrm{pm}) \wedge \operatorname{close}_{S}(Q,(\lambda e . \tau(e) \subseteq 3 \mathrm{pm})) \wedge Q(e)\right]$

b. $[[$ Gianni è arrivato ]] $=\lambda e$. [arrive $(e, \operatorname{Gianni}) \wedge \operatorname{Past}(e)]$

c. $[[$ Gianni è arrivato quasi alle tre $]]=\lambda e$. $[\operatorname{arrive}(e, \operatorname{Gianni}) \wedge \operatorname{Past}(e) \wedge$ $\neg \tau(e) \subseteq 3 \mathrm{pm} \wedge \exists Q_{<\mathrm{E}, \mathrm{t}} \in S\left[Q<_{S}(\lambda e . \tau(e) \subseteq 3 \mathrm{pm}) \wedge \operatorname{close}_{S}(Q,(\lambda e\right.$. $\tau(e) \subseteq 3 \mathrm{pm})) \wedge Q(e)]$

\footnotetext{
${ }^{6}$ A rigori, the equation (7) is not a lexical entry, as ' $\gamma$ ' here is a variable over semantic types, not the name of any particular type. This is in accordance to our assumption of the cross-categorial status of quasi.

${ }^{7}$ In other words, our assumption is that the relevant scale of alternatives to [[ alle tre ] is determined by a linear order which is defined on the basis of the natural relation of temporal precedence between times.
} 
[[ Gianni è arrivato quasi alle tre ]] $=1$ iff

iff $\exists e$ [arrive $(e$, Gianni $) \wedge \operatorname{Past}(e) \wedge \neg \tau(e) \subseteq 3 \mathrm{pm} \wedge \exists Q_{<\mathrm{E}, \downarrow} \in S\left[Q<_{S}(\lambda e\right.$. $\left.\tau(e) \subseteq 3 \mathrm{pm}) \wedge \operatorname{close}_{s}(Q,(\lambda e . \tau(e) \subseteq 3 \mathrm{pm})) \wedge Q(e)\right]$

Our analysis correctly predicts that sentence (8) is true if and only if there is a past event of Gianni's arrival which occurred before $3 \mathrm{pm}$, at a time which was close to $3 \mathrm{pm}$ (in a contextually relevant sense of being close).

\section{The Puzzle Revisited}

\subsection{Asymmetry between prima and dopo}

Following Del Prete (2008), we assume that prima is a temporal comparative with the same semantic analysis as più presto 'earlier', and that quasi underlyingly modifies the comparative morpheme più 'more' in $(1 \mathrm{a}, \mathrm{b}) .{ }^{8}$ On the other hand, we assume that dopo is not a temporal comparative, but an atomic predicate denoting the relation of temporal succession between events.

Notice that if dopo were a comparative with the meaning of più tardi 'later', we would expect modification by quasi to be possible. For example, sentences $(13 a, b)$, which differ from $(2 a, b)$ in having the comparative più tardi in the place of dopo, are perfectly acceptable: ${ }^{9}$

a. Le pere Cocomerine cadono quasi più tardi della fine dell'autunno.

'Cocomerine pears fall almost later than the autumn's end.'

b. L'avventura olimpica di Tommaso Rocchi è cominciata quasi più tardi della fine delle Olimpiadi.

'The Olympic adventure of $T$. Rocchi started almost later than the Olympics' end.'

The contrast between (14) and (15) shows that prima patterns with più presto when it is modified by quasi, while dopo does not pattern with più tardi.

\footnotetext{
${ }^{8}$ For a detailed exposition of the analysis of prima which is presupposed here, see Del Prete (2008: 174-85).

${ }^{9}$ The comparative più tardi does not allow for clausal complements like the ones that the temporal connective dopo takes in $(2 \mathrm{a}, \mathrm{b})$. For this reason, the examples $(13 \mathrm{a}, \mathrm{b})$ are constructed with nominal complements instead of clausal ones. This syntactic difference between $(2 \mathrm{a}, \mathrm{b})$ and $(13 \mathrm{a}$, b), however, does not bear on the contrast of acceptability between the two pairs which is highlighted in the main text. For one thing, sentences (i) and (ii), which are obtained via nominalization of the temporal clause from (2a) and (2b), respectively, are also unacceptable on the intended reading:

(i) ? Le pere Cocomerine cadono quasi dopo la fine dell'autunno.

(ii) ? L'avventura olimpica di Tommaso Rocchi è cominciata quasi dopo la fine delle Olimpiadi.
} 
(14) [Context: Maria always arrives too early at a party, when the person throwing the party is still setting things up. Today, Pietro arrived only two minutes later than Maria (who arrived too early again).]

Pietro è arrivato quasi prima di Maria / quasi più presto di Maria.

'Pietro arrived almost before Maria / almost earlier than Maria.'

(15) [Context: Maria is always late, no matter what. Today there was a meeting with Maria, Pietro and Samuele. Pietro arrived late, only two minutes before Maria arrived (and she was late again).]

Pietro è arrivato *quasi dopo Maria / quasi più tardi di Maria.

'Pietro arrived almost after Maria / almost later than Maria.'

\subsection{Analysis of quasi prima}

To exemplify our analysis of quasi prima, we focus on sentence (1a), repeated as (16) below in a simplified form. ${ }^{10}$ From the assumption that quasi underlyingly modifies più in (16), and that the meaning of più is the relation between sets of degrees given in (17), it follows that the scalar alternatives relevant to the interpretation of (16) are also relations between sets of degrees, such as are represented in $(18 \mathrm{a}, \mathrm{b})$. We assume that the relevant scale is [[a bit less $]]<_{S}[[$ as much as ]] $<_{S}[[$ more ]]. Accordingly, (18a) gives the semantic value of the comparative marker a bit less, while (18b) gives the semantic value of the equative marker as much as.

(16) Le pere Cocomerine vengono consumate quasi prima che cadano.

'Cocomerine pears are consumed almost before they fall.'

$$
[[\text { più }]]=\lambda P_{<\mathrm{d}, \downarrow} \cdot \lambda Q_{<\mathrm{d}, \downarrow} . \exists d[\neg P(d) \wedge Q(d)]
$$

a. $\lambda P_{<\mathrm{d}, \triangleright .} . \lambda Q_{<\mathrm{d}, \triangleright .} . \exists d\left[P(d) \wedge \neg Q(d) \wedge \exists d^{\prime}\left[d^{\prime} \leq d \wedge \operatorname{close}\left(d^{\prime}, d\right) \wedge Q\left(d^{\prime}\right)\right]\right]$

b. $\lambda P_{<\mathrm{d}, \triangleright\rangle} . \lambda Q_{<\mathrm{d}, \triangleright>} . \forall d[P(d) \rightarrow Q(d)]$

The main steps of the compositional derivation of (16)'s truth-conditions are reported in (19a-d).

a. $\quad[[$ quasi più $]]=\lambda P_{<\mathrm{d}, \mathrm{t}>} . \lambda Q_{<\mathrm{d}, \downarrow)} \neg \exists d[\neg P(d) \wedge Q(d)] \wedge \exists \tau_{<<\mathrm{d}, \downarrow,<<\mathrm{d}, \downarrow, \downarrow)>}$ $\in S\left[\mathcal{T}<_{S}\left(\lambda P_{<\mathrm{d}, \downarrow} \cdot \lambda Q_{<\mathrm{d}, \triangleright \triangleright} . \exists d[\neg P(d) \wedge Q(d)]\right) \wedge \operatorname{close}_{S}\left(\mathcal{T},\left(\lambda P_{<\mathrm{d}, \downarrow}\right.\right.\right.$. $\left.\left.\left.\lambda Q_{<\mathrm{d}, \mathrm{t}} . \exists d[\neg P(d) \wedge Q(d)]\right)\right) \wedge \mathcal{T}(P, Q)\right]$

b. [[ than Cocomerine pears fall early ]] $=\lambda d$. $\exists e[$ fall $(e$, C-pears $) \wedge$ early $(e, d)]$

c. $\quad[[$ Cocomerine pears are consumed early $]]=\lambda d . \exists e[\operatorname{consumed}(e, \mathrm{C}$ pears) $\wedge \operatorname{early}(e, d)]$

\footnotetext{
${ }^{10}$ From the original, naturally occurring example (1a) we omit the modal of necessity expressed by the auxiliary verb vanno ('go' = 'have to'), as this is irrelevant to the part of the semantic interpretation of (1a) that we are concerned with.
} 
d. [[ quasi più [than Cocomerine pears fall early] [Cocomerine pears are consumed early] ] $=1$ iff iff $\neg \exists d[\neg \exists e[$ fall $(e, \mathrm{C}$-pears $) \wedge$ early $(e, d)] \wedge \exists e[\operatorname{consumed}(e, \mathrm{C}$ pears $) \wedge$ early $(e, d)]] \wedge \exists \mathcal{T}_{<<\mathrm{d}, \downarrow,<<\mathrm{d}, \downarrow, \downarrow)>} \in S\left[\mathcal{T}<_{S}\left(\lambda P_{<\mathrm{d}, \downarrow>} . \lambda Q_{<\mathrm{d}, \downarrow)} . \exists d\right.\right.$ $[\neg P(d) \wedge Q(d)]) \wedge \operatorname{close}_{S}\left(\mathcal{T},\left(\lambda P_{<\mathrm{d}, \triangleright\rangle} . \lambda Q_{<\mathrm{d}, \triangleright .} . \exists d[\neg P(d) \wedge Q(d)]\right)\right) \wedge$ $\mathcal{T}(\lambda d . \exists e[$ fall $(e, \mathrm{C}$-pears $) \wedge \operatorname{early}(e, d)], \lambda d . \exists e[$ consumed $(e, \mathrm{C}$-pears $)$ $\wedge$ early $(e, d)])]$

Given the above assumption about what the scale $S$ is in this case, (19d) says that sentence (16) is true iff Cocomerine pears are not consumed by a time at which they have not yet fallen, and either Cocomerine pears are consumed soon after they fall, or they are consumed at the same time as they fall. We may assume that the latter alternative is excluded on the basis of general world-knowledge, so that the alternative which remains open is that Cocomerine pears are consumed soon after they fall, which is what (16) intuitively conveys.

The analysis just proposed for quasi prima differs from our analysis of quasi alle tre (in Section 3) in one important respect. For the latter, we have assumed that the constituent modified by quasi is the entire temporal phrase alle tre, while for the former we have assumed that quasi modifies just the comparative morpheme underlying prima. The reason for this difference relies on our account of the meaning of quasi: as long as quasi finds a constituent $\mathrm{X}$ that denotes a limit point on a natural scale, as is the case with più, the semantic requirement of quasi is satisfied and quasi can modify $\mathrm{X}$ without need to further compose $\mathrm{X}$ with other constituents. The semantic value of the locational temporal preposition at, on the other hand, does not belong to any natural scale, and, unlike più and prima, $a$ 'at' cannot be uttered in isolation (i.e. without its internal complement). Hence, it is only from the composition of the preposition with its internal argument that a set of scalar alternatives can be retrieved.

\subsection{The Gap: Why quasi dopo is Ruled Out}

We return now to the unacceptability of quasi dopo, as shown in $(2 \mathrm{~b})$, repeated below as (20):

(20) ? Le pere Cocomerine cadono quasi dopo che è passato l'autunno.

'Cocomerine pears fall almost after the autumn has passed.'

Following Del Prete (2008), we assume that dopo is not a temporal comparative; rather, its core semantic contribution is a binary relation of temporal succession between events. The lexical entry that we assume for dopo is given in (21) below. The first argument of the function which interprets dopo is instantiated by temporal measures, which we model as equivalence classes of time intervals in an obvious way: two intervals are members of the same temporal measure iff they have the same size. The first argument gives the measure of the interval $\tau\left(e_{2}\right)-$ 
$\tau\left(e_{1}\right)$ which separates $\tau\left(e_{2}\right)$ from $\tau\left(e_{1}\right) .{ }^{11}$ The functor ' $\mu$ ' in (21) denotes a function which maps any time interval onto its temporal measure. We assume that when the measure-argument $s$ is not bound by an overt Measure Phrase, its value is provided by the extra-linguistic context.

$$
[[\text { dopo }]]=\lambda s . \lambda e_{1} \cdot \lambda e_{2} \cdot \tau\left(e_{2}\right)>_{\mathrm{I}} \tau\left(e_{1}\right) \wedge \mu\left(\tau\left(e_{2}\right)-\tau\left(e_{1}\right)\right) \geq s^{12}
$$

On the basis of this analysis, it is not clear what could be the scale that the semantic value of dopo could be part of, and what could be the set of alternatives to [[ dopo ]] that would be ranked lower on that scale. ${ }^{13}$ We conclude that the semantics of dopo fails to provide the $f$-pair that quasi takes as first argument, hence dopo does not satisfy the selectional restrictions of quasi. ${ }^{14}$

Notice that there are naturally occurring sentences in which quasi precedes dopo, as in $(22 \mathrm{a}, \mathrm{b})$, adapted from examples found in the web:

a. Il pubblico romano si mostra affettuosissimo con numerosi applausi quasi dopo ogni canzone.

'The Roman audience shows itself very affectionate by means of many applauses almost after every song.'

b. Quasi dopo trenta giorni ho fatto un test di gravidanza ed è risultato negativo.

'Almost after thirty days I did a pregnancy test and it was negative.'

We claim that in these examples, in spite of the adverb immediately preceding the temporal connective, the modification relation is not between quasi and dopo, but

\footnotetext{
${ }^{11}$ An interval $i_{1}$ separates two intervals $i_{2}, i_{3}$ if and only if $i_{2} \cap i_{3}=\varnothing$ and $i_{1}$ is the minimal interval $i^{*}$ such that $\left(i^{*} \cup\left(i_{2} \cup i_{3}\right)\right)$ is an interval.

${ }^{12}$ In this lexical entry, the relation $>_{I}$ is a strict partial order defined over the set of time intervals $\mathrm{I}$, and it satisfies the following condition $\left(>_{\mathrm{T}}\right.$ is the strict total order of temporal succession defined over the set of time instants $\mathrm{T}$ ):

(i) $i>_{\mathrm{I}} i^{\prime}$ iff $\forall x \forall y\left[x \in i \wedge y \in i^{\prime} \rightarrow x>_{\mathrm{T}} y\right]$

${ }^{13}$ In other words, the meaning of dopo does not satisfy the Condition on Limit Points (cf. footnote 6): it does not contribute to the truth conditions of the sentence a definite value $v$ on a scale that would allow the speaker to recover a value ranked lower than $v$ and close to it. In this respect, dopo behaves in a similar way to qualche 'some': $A$ dopo $B$ can be true as long as the run time of the A-eventuality follows the run time of the B-eventuality, but there could be any non-null distance between the two run times.

${ }^{14}$ There are other contrasts, besides the puzzle discussed in this paper, that show that dopo, unlike prima, does not lend itself to a scalar interpretation. One such contrast involves modification by the scalar adverb ancora 'still', 'even'. As shown in (i) and (ii), while ancora prima can be interpreted as 'even earlier', ancora dopo does not have the mirroring interpretation 'even later', and the only possible reading of (ii) is the one expressed by its English translation below. In (ii) ancora gets the non-scalar meaning 'again' and modifies the VP of the main clause, not the temporal connective dopo (see Del Prete 2008: 166-67 for discussion of this contrast).

(i) Gianni si è svegliato ancora prima del sorgere del sole.

'Gianni woke up even earlier than the sunrise.'

(ii) Gianni si è addormentato ancora dopo le due del mattino.

'Gianni fell asleep again after 2 AM.'

(unacceptable on the reading 'Gianni fell asleep even later than 2 AM.')
} 
rather between quasi and the NP to the right of the temporal connective. In (22a), quasi intuitively modifies the quantified NP (QNP) ogni canzone 'every song', which is the complement of dopo. This sentence has a distributive interpretation: almost every song is such that many applauses followed it (see Amaral \& Del Prete [2009] for a full analysis). The contribution of quasi can be analyzed according to the meaning components identified above: (22a) entails the negation of the prejacent (it is not true that the Roman audience shows itself affectionate by means of applauses after every song), and it entails the existential quantification over lower ranked alternatives (for some quantifier $Q$ which approximates the strength of the universal quantifier, the Roman audience shows itself affectionate by means of applauses after $Q$ song). The claim that the modifier-modifiee relation holds between quasi and the QNP can be tested as follows: if we replace the universal NP in (22a) with the NP molte canzoni 'many songs', as in (23), we obtain an anomalous sentence, in which quasi remains uninterpretable.

(23) ?Il pubblico romano si mostra affettuosissimo con numerosi applausi quasi dopo molte canzoni.

'? The Roman audience shows itself very affectionate by means of many applauses almost after many songs.'

Indeed, while quasi can modify universal NPs, it cannot modify NPs with the determiner molti: note the contrast between the good sentence Ho ascoltato quasi ogni canzone 'I have listened to almost every song' and the deviant sentence Ho ascoltato quasi molte canzoni 'I have listened to almost many songs'. If quasi is removed from (23), a perfectly acceptable sentence results. As for (22b), quasi modifies the Measure Phrase (MP) trenta giorni 'thirty days', which occurs to the right of dopo. Accordingly, we have the interpretation that a period of almost thirty days had passed (from some contextually salient event), when the subject did a pregnancy test. The interpretation of quasi can be spelled out according to our analysis: (22b) entails the negation of the prejacent (it is not true that the subject did a pregnancy test after thirty days) and the existential quantification over lower ranked alternatives (for some numerical value $n$ which is smaller than the number thirty and close to it, the subject did a pregnancy test after $n$ days). The same test, crucial for the claim that quasi does not bear a relation of modification to dopo in (22a), can be applied to (22b): if we replace the MP in (22b) with diversi giorni 'several days', the result is the deviant sentence (24):

? Quasi dopo diversi giorni ho fatto un test di gravidanza ed è risultato negativo.

'? Almost after several days I did a pregnancy test and it was negative.'

While quasi can modify phrases like trenta giorni 'thirty days', it cannot modify a phrase with the determiner diversi 'several'. This is shown by the contrast between the good sentence Ho lavorato per quasi trenta giorni 'I worked for almost thirty days' and the anomalous sentence Ho lavorato per quasi diversi giorni 'I worked for almost several days'. Notice that if we remove quasi from (24), the sentence becomes perfectly acceptable. 
In (22b), the internal event-argument of dopo is provided by the context: the sentence is interpreted in context $c$ as meaning that the speaker did a pregnancy test thirty days after the event $e$ occurred $-e$ being a salient event in $c$. On our analysis of (22b), quasi modifies the MP trenta giorni 'thirty days' to the right of the temporal connective. We assume that the modified MP realizes the measure-argument of dopo, and propose a semantics for MPs like trenta giorni in which they denote generalized quantifiers over temporal measures. Following Heim and Kratzer (1998), phrases denoting generalized quantifiers are taken to undergo Quantifier Raising. The interpretation of trenta giorni is given in (27), where ' $\mathrm{M}$ ' denotes the type of temporal measures and ' $\mathrm{s}$ ' is a variable of type M.

$$
\text { [[ thirty days ]] }=\lambda P_{<\mathrm{M}, \triangleright \triangleright} . \exists s[P(s) \wedge s \geq 30 \text {-days }]
$$

We assume that quasi and trenta giorni form a discontinuous constituent [MP quasi [MP trenta giorni]] in (22b), which denotes a generalized quantifier over temporal measures. As such, it undergoes $\mathrm{QR}$, the result being a semantic representation like (28). The truth-conditions of (22b) are derived in (29a-d).

\section{[Almost [thirty days] $]_{1} 1\left[\mathrm{I}\right.$ did a pregnancy test after $\left.t_{1} e^{*}\right]$}

a. [[ almost thirty days ]] $=\lambda P_{<\mathrm{M}, \triangleright .} \neg \exists s[P(s) \wedge s \geq 30$-days $] \wedge$ $\exists \wp<<\mathrm{M}, \triangleright, \downarrow) \in S\left[\wp<<_{\mathrm{S}} \lambda P_{<\mathrm{M}, \downarrow} . \exists s[P(s) \wedge s \geq 30\right.$-days $] \wedge \operatorname{close}_{s}(\wp$, $\lambda P_{<\mathrm{M}, \triangleright} . \exists s[P(s) \wedge s \geq 30$-days $\left.\left.]\right) \wedge \wp(P)\right]$

b. $\left[\left[\right.\right.$ after $\left.\left.t_{1} e^{*}\right]\right]=\lambda e_{2} . \tau\left(e_{2}\right)>\tau\left(e^{*}\right) \wedge \mu\left(\tau\left(e_{2}\right)-\tau\left(e^{*}\right)\right) \geq \mathrm{g}\left(t_{1}\right)$

c. [[ $1\left[\mathrm{I}\right.$ did a pregnancy test after $\left.\left.\left.t_{1} e^{*}\right]\right]\right]=\lambda \mathrm{s}$. $\exists e$ [I-did-pregnancytest $\left.(e) \wedge \tau(e)>\tau\left(e^{*}\right) \wedge \mu\left(\tau(e)-\tau\left(e^{*}\right)\right) \geq s\right]$

d. [[ (28) ]] $=1$ iff $\neg \exists s\left[\exists e\left[\right.\right.$ [I-did-pregnancy-test $(e) \wedge \tau(e)>\tau\left(e^{*}\right) \wedge$ $\left.\mu\left(\tau(e)-\tau\left(e^{*}\right)\right) \geq s\right] \wedge s \geq 30$-days] $\wedge \exists \wp<<\mathrm{M}, \triangleright, \downarrow \in S\left[\wp<\mathrm{s} \lambda P_{<\mathrm{M}, \triangleright)} . \exists s\right.$ $[P(s) \wedge s \geq 30$-days $] \wedge \operatorname{close}_{s}\left(\wp, \lambda P_{<\mathrm{M}, \triangleright} . \exists s[P(s) \wedge s \geq 30\right.$-days $\left.]\right) \wedge$ $\wp\left(\lambda s . \exists e\right.$ [I-did-pregnancy-test $(e) \wedge \tau(e)>\tau\left(e^{*}\right) \wedge \mu\left(\tau(e)-\tau\left(e^{*}\right)\right) \geq$ $s])]$

Assuming that the alternatives to trenta giorni form a scale like in (30), in which the ordering is determined by the measure of the time interval, in such a way that lower ranked alternatives have time intervals of smaller size, we can rewrite the truth conditions for (22b) in the simplified form given in (31).

$$
\begin{aligned}
& \lambda P_{<\mathrm{M}, \triangleright .} . \exists s[P(s) \wedge s \geq 28 \text {-days }]<_{s} \lambda P_{<\mathrm{M}, \downarrow} . \exists s[P(s) \wedge s \geq 29 \text {-days }]<_{s} \\
& \lambda P_{<\mathrm{M}, \triangleright .} . \exists s[P(s) \wedge s \geq 30 \text {-days }] \\
& \neg \exists s\left[\exists e \text { [I-did-pregnancy-test }(e) \wedge \tau(e)>\tau\left(e^{*}\right) \wedge \mu\left(\tau(e)-\tau\left(e^{*}\right)\right) \geq s\right] \wedge s \geq \\
& \text { 30-days] } \wedge \exists n n<30 \wedge \operatorname{close}(n, 30) \wedge \exists s[\exists e \text { [I-did-pregnancy-test }(e) \wedge \\
& \left.\tau(e)>\tau\left(e^{*}\right) \wedge \mu\left(\tau(e)-\tau\left(e^{*}\right)\right) \geq s\right] \wedge s \geq n \text {-days] }
\end{aligned}
$$


Our analysis predicts that sentence (22b) is true if and only if (a) there is no temporal measure $s$ at least as great as 30 days such that an event of the speaker doing a pregnancy test follows the contextually relevant event $e^{*}$ by the measure $s$, and (b) there is a temporal measure $s$ a little bit less than 30 days such that an event of the speaker doing a pregnancy test follows the contextually relevant event $e^{*}$ by the measure $s$.

In conclusion, on our account the acceptability of examples $(22 \mathrm{a}, \mathrm{b})$ is explained by the presence, in the complement of dopo, of a lexical item that denotes a limit point on a scale and hence may combine with quasi. On the other hand, the semantics of dopo does not meet the selectional restrictions of quasi: the relation of temporal succession itself which dopo denotes is not a limit point on a natural scale. On this account, the unacceptability of *quasi dopo belongs to the same type of semantic anomalies as *quasi sporco 'almost dirty' and *quasi qualche 'almost some', that have been discussed in detail in the literature on almost (Hitzemann 1992, Morzycki 2001, Penka 2006).

We briefly address now the reading of quasi dopo in which quasi seems to mean 'a little bit', mentioned in Section 1 (the "shortly after" reading). Sentence $(2 \mathrm{a})$, repeated here as $(32)$, is a relevant example:

(32) Le pere Cocomerine cadono quasi dopo che è passato l'autunno.

'Cocomerine pears fall shortly after the autumn has passed.'

The "shortly after" reading of (32) is prima facie problematic for our analysis of quasi: on this reading (32) entails that Cocomerine pears fall after the autumn has passed, so the polar component is apparently absent in this case. Our proposal is that the "shortly after" reading of (32) is obtained by taking quasi to be modifying the underlying measure-argument of dopo, whose contextually provided value is zero. Notice that the measure-argument cannot have a negative value, hence the ordering of the alternatives must be from higher to lower measures bounded by zero. Thus, the reading obtained for (32) without quasi is that Cocomerine pears fall immediately after the autumn has passed, and the reading obtained for (32) is that Cocomerine pears fall almost immediately after the autumn has passed. On this account, the semantic contribution of quasi to the "shortly after" reading of (32) is exactly the same as we have described and formalized in Section 3: the reading at issue indeed corresponds to the paraphrase 'The falling of Cocomerine pears does not follow the end of the autumn by a measure of zero, but it follows the end of the autumn by a measure which approximates zero', which makes it clear that the polar and the proximal component are both present.

\section{Modification of Comparatives by quasi}

The analysis proposed in Section 4.2 predicts that there is no truth-conditional equivalence between $x$ è quasi più $P$ di $y$ ' $x$ is almost P-er than $y$ ' and $x$ è quasi tanto $P$ quanto $y$ ' $x$ is almost as much $\mathrm{P}$ as $y$ '. The compositional analysis of these two constructions shows that the limit points are different in the two cases: the 
limit point is the semantic value of the comparative morpheme più in the former, and the semantic value of the equative morpheme tanto quanto in the latter. Hence, in the case of $x$ is almost P-er than $y$ the scalar alternatives that are ranked lower than the limit point include at least one alternative, the semantic value of the equative, which is excluded from the interpretation of $x$ is almost as much $P$ as $y$ due to the polar component of quasi. This is shown formally in (33a, b):

a. $[[x$ is almost $P$-er than $y]]=1$ iff

(i) $\neg \exists d[\neg \mathrm{P}(y, d) \wedge \mathrm{P}(x, d)]$ (polar component)

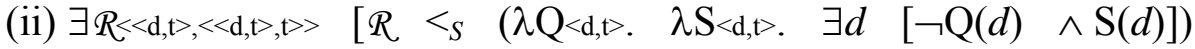
$\wedge \operatorname{close}_{S}(R,(\lambda \mathrm{Q} . \lambda \mathrm{S} . \exists d[\neg \mathrm{Q}(d) \wedge \mathrm{S}(d)])) \wedge R(\lambda d . \mathrm{P}(y, d))(\lambda d . \mathrm{P}(x$, d))] (proximal component)

b. $[[x$ is almost as much $P$ as $y]]=1$ iff

(i) $\neg \forall d[P(y, d) \rightarrow P(x, d)]$ (polar component)

(ii) $\exists \mathbb{R}<<\mathrm{d}, \mathrm{t},<<\mathrm{d}, \downarrow, \downarrow)>[R<(\lambda Q<\mathrm{d}, \downarrow$. $\lambda S<\mathrm{d}, \mathrm{R} . \forall d[Q(d) \rightarrow S(d)]) \wedge \operatorname{close}(\mathbb{R}$, $(\lambda Q . \lambda S . \forall d[Q(d) \rightarrow S(d)])) \wedge R(\lambda d . \mathrm{P}(y, d))(\lambda d . \mathrm{P}(x, d))]$ (proximal component)

Despite this truth-conditional difference, we observe that the modified comparative (quasi più) is systematically used in scenarios in which the selected scalar alternative is a "less than" relation (a lower ranked alternative to the equative form), and not the "as much as" relation.

Consider the interpretation of quasi più alto ('almost higher than') and quasi più vicino ('almost closer than') ${ }^{15}$ in the following naturally occurring examples:

a. La città dovrebbe mantenere la propria identità ed a tale proposito è facile intuire che un grattacielo di 40 piani, ben 120 metri di altezza, quasi più alto del Pirelli e della Madonnina, ${ }^{16}$ costruito in quella zona potrebbe alterare irreparabilmente l'equilibrio delle altimetrie del quartiere.

'The city should preserve its own identity, and in this connection it's easy to imagine that a 40 floors, 120 meters high skyscraper, almost higher than the Pirelli and the Madonnina, if built in that area, could irreparably alter the equilibrium of the altimetries of the district.'

\footnotetext{
${ }^{15}$ The modified comparative of adjectives like alto 'tall' and vicino 'close' is a good test case for our purposes as its use is sometimes accompanied by numerical estimates of the compared objects' height and distance values, and this makes it easier to determine the truth-conditional meaning of these expressions. Moreover, even if no numerical value is given in the discourse context, it is often clear from our world knowledge what the relation actually is between the heights or distances of the compared objects and locations.

${ }^{16}$ The Madonnina is a statue of the Virgin Mary that stands on top of the Duomo (Cathedral) of Milan. According to Wikipedia, "By tradition, no building in Milan is higher than the Madonnina. When Gio Ponti's Pirelli Building was being built in the late 1950's, at a height of $127.1 \mathrm{~m}$, a smaller replica of the Madonnina was placed atop the Pirelli building, so the new Madonnina remains the tallest point in Milan." In our analysis, we assume that the relevant standard of comparison is the Madonnina statue on top of the Pirelli building.
} 
b. È un paesino Grumolo delle Abbadesse, ${ }^{17}$ in provincia di Vicenza, ma quasi più vicino a Padova...

'It is a little village, Grumolo delle Abbadesse, in the Vicenza province but almost closer to Padova...'

In $(34 a, b)$ the modified comparative is true of a scenario where the converse of the comparative relation denoted by $x$ è più $P$ di $y$ holds: in (34a) $x$ è quasi più alto di $y$ is understood as meaning that the $120 \mathrm{~m}$ high skyscraper would be a little bit less high than the Madonnina, and in (34b) $x$ è quasi più vicino di $y$ is understood as meaning that the village Grumolo delle Abbadesse is a little bit less far from Vicenza than it is far from Padova. This fact parallels what we have previously observed about the interpretation of quasi prima. Note that in $(34 \mathrm{a}, \mathrm{b})$, the use of the modified equative (quasi tanto alto quanto and quasi tanto vicino quanto) would be felicitous and would yield an interpretation in which the selected scalar alternative would be "less than". According to our analysis, a "less than" relation is not ruled out as a possible interpretation of the modified comparative, as it is an alternative which is ranked lower than the più comparative relation on the relevant scale. However, on purely semantic terms this interpretation is surprising: the intended meaning of quasi( $x$ ̀̀ più $P$ di $y)$ corresponds to the same semantic alternative which would be obtained by the interpretation of quasi as a modifier of the equative.

We propose that the similarity of meaning in context between $x$ is almost $P$-er than $y$ and $x$ is almost as much $P$ as $y$ requires a pragmatic explanation. Whereas modification of the equative form by quasi is felicitous and interpretable in an out-of-the-blue context, the use of quasi( $x$ è più $P$ di $y$ ) is not felicitous in such a context. To exemplify: you can compare the heights of two people $x, y$ whose pictures have just been shown to you and to your interlocutor by saying $x$ is almost as tall as $y$, and this would be true as long as $x$ is a bit less tall than $y$. However, it would be strange for you to say in the same situation $x$ is almost taller than $y$. The use of quasi( $x$ è più $P$ di $y$ ) is restricted by certain contextual constraints. In other words, the set of contexts in which the modified comparative can be felicitously used is a proper subset of the set of contexts in which the modified equative can be used. We spell out these contextual constraints in (35):

Contextual restrictions on the use of quasi( $x$ è più $P$ di $y$ ).

$(\alpha)$ There is a contextually-shared assumption that in quasi( $x$ è più $P$ di $y$ ) the standard of comparison $y$ is $\mathrm{P}$ to a higher degree than $x$ in a contextually determined sub-scale of property P. This assumption can be generated either in the local context (it can be specific to the common ground of a community of speakers or to the epistemic or deontic models of the participants in a conversation, as in [34a]), or it can be generated in

\footnotetext{
${ }^{17}$ The village Grumolo delle Abbadesse is ca. $20 \mathrm{~km}$ away from Vicenza and ca. $28 \mathrm{~km}$ away from Padova, the capital cities of the respective provinces. Thus, the village is closer to Vicenza than to Padova. A map of the Vicenza province shows that Grumolo delle Abbadesse is very close to the border between the two provinces, hence the perception that it is close to the province of Padova.
} 
the global context on the basis of world knowledge (e.g. what we know about regional provinces, as in [34b]).

$(\beta)$ The unmodified comparative $x$ è più $P$ di $y$ expresses a relation which is not expected by the speaker. In other words, under normal circumstances, the speaker would expect a relation to hold between $x$ and $y$ which is the converse of the one expressed by $x$ è più $P$ di $y$.

These contextual restrictions are met in the contexts of utterance of the examples above, as shown in (36) for (34a) and in (37) for (34b):

(36) ( $\alpha$ ) In the deontic model of the speaker of (34a), the standard of comparison, the Madonnina statue, is higher than any other point in the Milan skyline, and hence is higher than the new skyscraper. Importantly, in (34a) it is not the particular statue called "Madonnina" which is considered for its actual height, but rather the individual concept associated with that name, which is expected to apply to the highest point in Milan.

( $\beta$ ) The unmodified comparative un grattacielo di 40 piani, ben 120 metri di altezza, più alto della Madonnina expresses a relation which is not expected by the speaker, given $(\alpha)$.

(37) ( $\alpha$ ) In (34b), the comparison is established between the degree to which Grumolo delle Abbadesse is close to Padova and the degree to which it is close to Vicenza (which is the standard of comparison). Crucially, Vicenza and Padova are not being considered for their individual properties, but rather as capitals of provinces. The contextually-shared assumption is that, prototypically, a village or town is closer to the capital of the province it belongs to than to the capital of a different province.

$(\beta)$ The unmodified comparative Grumolo delle Abbadesse è un paesino più vicino a Padova che a Vicenza expresses an unexpected relation.

Recall that in the factual context of (34a), the height of the new skyscraper is $120 \mathrm{~m}$, a value which is lower than the height of the Madonnina statue on top of the Pirelli. However, the degree to which the new building would be high approximates the value of the Madonnina's height to such an extent that their difference is of no significance for the purposes of the conversation. Hence, the intended meaning of the modified comparative in (34a) is that the new building would be too high. As for (34b), the intended meaning of the modified comparative is that Grumolo delle Abbadesse is closer to Padova than one would expect, given that the village belongs to the province of Vicenza. Again, the actual difference between the degree to which Grumolo delle Abbadesse is close to Padova and the degree to which it is close to Vicenza is contextually irrelevant since it is very small.

The restrictions presented in (35) are the same that govern felicitous utterances of quasi prima. In (38) and (39), we provide a stepwise application of 
the schema (35) to examples (1a) and (1b) above, respectively. Given our analysis of prima, the property that instantiates $P$ in (35) is the property of being early.

(38) ( $\alpha$ ) According to world knowledge, under normal circumstances fruit is consumed after it has been picked from the tree. Hence, the contextuallyshared assumption crucial for this example is that the standard of comparison, which is the event of Cocomerine pears being picked, is early to a higher degree than the degree to which the event of the pears being consumed is early.

$(\beta)$ Thus, the unmodified comparative Le pere Cocomerine vengono consumate prima che cadano expresses a relation that is not expected by the speaker.

As in examples $(34 \mathrm{a}, \mathrm{b})$ with the overt comparatives, the relation between the events described in (1a) is that the event of the pears being consumed is $a$ bit less early than the event of the pears being picked. However, the minimal difference between these degrees of earliness underlies the use of the modified comparative: the intended meaning of (1a) is that Cocomerine pears, since they are extremely perishable, must be consumed very early.

(39) ( $\alpha$ ) The standard of comparison $y$, the beginning of Rocchi's adventure at the Olympics, is early to a high degree relative to the running time of the Olympics. The expectation provided by the global context is that the ending event $x$ of any Olympic adventure should be less early than the beginning event of the same adventure (beginnings precede endings!).

$(\beta)$ The unmodified comparative L'avventura è finita prima di cominciare expresses a relation which is not expected by the speaker.

In (1b), the ending of Rocchi's adventure is only a bit less early than the beginning of the adventure. For the expected duration of an Olympic adventure, the actual difference between the two degrees of earliness is irrelevant. The intended meaning of the modified comparative in (1b) is that the ending of Rocchi's adventure happened too early, i.e. earlier than expected.

\section{Conclusion}

In this paper, we have proposed a solution for the asymmetric interpretation of quasi prima and quasi dopo. This solution sheds light on the sensitivity of quasi to scale structure: while the meaning of prima provides the scalar alternatives and the limit point required by the semantics of quasi, the relation of temporal succession between events contributed by dopo cannot be understood as a limit point on a scale. Modification by quasi provides further evidence for a separate semantic analysis of prima and dopo. 
Finally, building on an analysis of prima as a temporal comparative, we have identified a general pattern of interpretation of quasi with comparative expressions, which includes both a semantic and a pragmatic component.

\section{References}

Amaral, Patrícia: 2007, The meaning of approximative adverbs: Evidence from European Portuguese. Ph.D. dissertation, OSU.

Amaral, Patrícia: to appear, 'Entailment, assertion, and textual coherence: The case of almost and barely' (to appear in Linguistics).

Amaral, Patrícia and Fabio Del Prete: 2009, 'Approximating the Limit: the Interaction between quasi 'almost' and Some Temporal Connectives in Italian', paper under review.

Davidson, Donald: 1967, 'The logical form of action sentences', in N. Rescher (ed.), The Logic of Decision and Action, 81-120. Pittsburgh: University of Pittsburgh Press.

Del Prete, Fabio: 2008, 'A Non-Uniform Semantic Analysis of the Italian Temporal Connectives Prima and Dopo', Natural Language Semantics 16, 157-203.

Heim, Irene and Angelika Kratzer: 1998, Semantics in Generative Grammar, Malden \& Oxford: Blackwell.

Hitzeman, Janet: 1992, 'The Selectional Properties and Entailments of "Almost", in C. P. Canakis, G. P. Chan, and J. Marshall Denton (eds.), Papers from the $28^{\text {th }}$ Regional Meeting of the Chicago Linguistic Society, 225-238.

Horn, Laurence R.: 1972, On the Semantic Properties of Logical Operators in English. Doctoral Dissertation, UCLA.

Landman, Fred: 1991, Structures for Semantics, Dordrecht: Kluwer Academic Publishers.

Landman, Fred: 2000, Events and Plurality, Dordrecht: Kluwer Academic Publishers.

Morzycki, M.: 2001, 'Almost and Its Kin, Across Categories', in Proceedings of SALT XI, CLC Publications, Cornell University, Ithaca.

Parsons, Terence: 1985, 'Underlying events in the logical analysis of English', in E. LePore and B. McLaughlin (eds.), Actions and events: Perspectives on the philosophy of Donald Davidson, 235-267. Oxford: Basil Blackwell.

Parsons, Terence: 1990, Events in the semantics of English: A study in subatomic semantics, Cambridge: MIT Press.

Penka, Doris: 2006, 'Almost There: The Meaning of almost', in C. Ebert and C. Endriss (eds.), Proceedings of Sinn und Bedeutung 10, 275-286. Berlin: ZAS Papers in Linguistics 44.

Rooth, Maths: 1985, Association with Focus. Doctoral Dissertation, UMass Amherst.

Sevi, Aldo: 1998, A Semantics for Almost and Barely. M.A. thesis, Tel-Aviv University. 